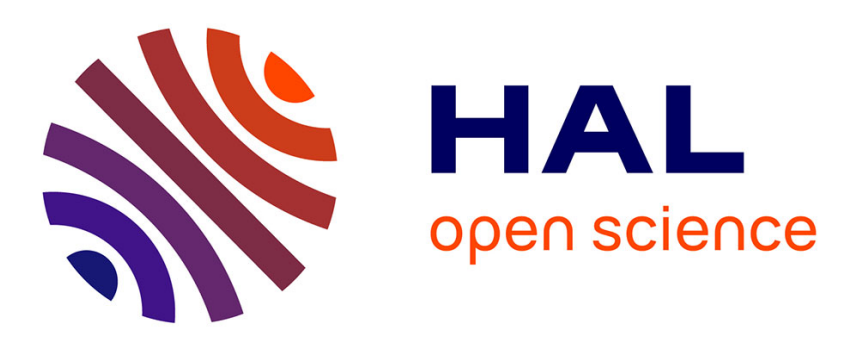

\title{
L'évolution des protestantismes en Corée du Sud : un rapport ambigu à la modernité
}

\author{
Nathalie Luca
}

\section{To cite this version:}

Nathalie Luca. L'évolution des protestantismes en Corée du Sud : un rapport ambigu à la modernité. Critique Internationale, 2004, 22, pp.111-124. 10.3917/crii.022.0111 . halshs-01821659

\section{HAL Id: halshs-01821659 \\ https://shs.hal.science/halshs-01821659}

Submitted on 23 Jul 2019

HAL is a multi-disciplinary open access archive for the deposit and dissemination of scientific research documents, whether they are published or not. The documents may come from teaching and research institutions in France or abroad, or from public or private research centers.
L'archive ouverte pluridisciplinaire HAL, est destinée au dépôt et à la diffusion de documents scientifiques de niveau recherche, publiés ou non, émanant des établissements d'enseignement et de recherche français ou étrangers, des laboratoires publics ou privés. 


\section{L'évolution des protestantismes en Corée du Sud:un rapport ambigu à la modernité}

par Nathalie Luca

Le succès du christianisme - et plus particulièrement des protestantismes - en Corée du Sud est d'autant plus étonnant que les deux grandes puissances qui l'entourent, la Chine et le Japon, sont restées plutôt indifférentes à cette religion. Depuis une dizaine d'années, on observe néanmoins, une stagnation dans la progression du nombre des chrétiens. Après une croissance explosive de $333,7 \%$ entre 1962 et 1970 , le taux de conversions est tombé à 23,9 \% entre 1985 et 1991 , puis à $1,4 \%$ entre 1991 et 19941. Par ailleurs, de récentes observations de terrain mettent en évidence la réserve actuelle, si ce n'est le retrait, des Églises protestantes. Les rues de Séoul et de chaque ville et village de la Corée du Sud n'en continuent pas moins d'être jalonnées de croix illuminées qui témoignent de ce que fut le prosélytisme de ces Églises jusqu'à la fin du XXe siècle.

Plusieurs étapes ont marqué l'évolution des Églises protestantes de la fin du XIXe siècle à nos jours. Leur succès a d'abord été lié au besoin de reconstruction d'une identité nationale mise à mal par quarante ans de colonisation japonaise. Durant la guerre de Corée (1950-1953), la Corée du Sud a utilisé la symbolique

1. Sung-Ho Kim, « Rapid Modernisation and the Future of Korean Christianity », Religion, 32, 2002, p. 27. Les données statistiques ne sont pas fournies pour les années 1970-1985. 
chrétienne du Bien et du Mal pour diaboliser le Nord communiste et présenter sa propre croissance économique comme le signe de son élection divine. Le développement accéléré des Églises protestantes conservatrices et progouvernementales s'est donc fait conjointement à la montée en puissance de la Corée du Sud sur la scène économique internationale. Mais le succès du protestantisme a joué un rôle ambigu dans la modernisation du pays. La résistance à la colonisation japonaise, puis le combat contre le communisme, dont ces Églises ont fait leurs thèmes de mobilisation, ont largement contribué à accroître leur influence, mais ont, en même temps, retardé les processus de sécularisation, d'individualisation et de désenchantement caractéristiques des sociétés modernes occidentales. Le lent processus de démocratisation entamé dans les années 1980, la récente politique de réunification et la crise asiatique de 1997 ont entraîné la stagnation de leur activité. Cette perte de vitesse a eu notamment pour conséquence d'amener les Coréens sur le terrain d'un nouvel individualisme identitaire.

L'analyse des facteurs explicatifs de la courbe en cloche du christianisme en Corée du Sud laissera ici de côté les activistes chrétiens, appartenant à l'Église catholique ou aux courants libéraux protestants - prêtres ouvriers, mouvements féministes, églises syndicalistes au service des plus démunis -, qui se sont développés à partir des années 1970 et plus encore durant les années 1980. Parmi ces mouvements, certains œuvraient sous la bannière de la théologie Minjung (théologie de la libération) qui entendait supprimer toute notion de classes sociales pour ne plus s'attacher qu'au désespoir de populations aussi bien coréenne qu'internationales, unies en une vaste communauté chrétienne par un même sentiment de souffrance2. En luttant contre les régimes dictatoriaux, ces courants contestataires chrétiens ont œuvré pour la démocratisation du pays et leur combat a certainement contribué à bâtir la Corée du Sud actuelle. Mais, fortement surveillés, voire réprimés par les gouvernements militaires, ils ont été freinés dans leur développement et ne sont donc pas une clé pour la compréhension de l'essor des mouvements se réclamant du christianisme.

\section{Les difficultés d'implantation et de développement du catholicisme}

À la fin de la seconde guerre mondiale, les chrétiens - toutes dénominations confondues - ne représentaient guère plus de $1 \%$ de la population coréenne et étaient tout aussi affaiblis que les courants religieux ou philosophiques autochtones. Les monastères bouddhistes étaient restés isolés dans les montagnes depuis l'avènement de la dynastie des Yi et l'instauration du néoconfucianisme (1392-1910)3. Ce dernier, élément constitutif de l'identité coréenne, avait, quant à lui, perdu sa place privilégiée dans les hauts lieux du pouvoir, le concours de recrutement des fonctionnaires ( $k w a g o$ ), instauré par les néoconfucéens, ayant été supprimé en 1894 
après l'arrivée des Japonais. Sans être réprimées, ces deux religions ont été, durant la colonisation, surveillées et mises au service du gouvernement japonais4.

Le catholicisme a été introduit en Corée à la fin du XVIIIe siècle par des lettrés coréens qui rapportaient de Chine des ouvrages consacrés aux sciences et aux religions de l'Occident. Son développement a été stoppé de façon brutale par l'interdiction imposée aux convertis par l'autorité religieuse de Rome de pratiquer les rites confucéens qui étaient la clé de voûte de l'ordre établi. Les catholiques ont été aussi accusés de conspirer avec les Occidentaux pour renverser le pouvoir confucéen en place. Des répressions sanglantes ont eu lieu, dont celle de 1866 a fait environ 8000 morts. Durant la colonisation japonaise (1910-1945), l'Église catholique, traumatisée par le souvenir de ces massacres, n'a pas soutenu les mouvements de résistance et n'a pas participé aux manifestations pour l'indépendance du 1er mars 1919, largement orchestrées par les protestants. Au contraire, comme le souligne Évelyne Chérel, «elle [s'est pliée] aux pressions exigeantes des autorités coloniales, devenant notamment l'un des relais de leur politique de mobilisation»5. Lors de sa libération, le peuple coréen n'a pas oublié de condamner vivement cette collaboration qu'il comparait à l'engagement manifeste des protestants en faveur des causes nationales. Enfin, après avoir soutenu en 1948 le premier président de la République coréenne, le méthodiste Syngman Rhee, dont elle approuvait les convictions anticommunistes et pro-américaines, l'Église catholique s'est progressivement éloignée du gouvernement en place, pour soutenir une figure centrale de l'opposition, le catholique Myôn Chang, qui sera nommé Premier ministre de la seconde République. Celleci, instaurée après la révolution étudiante d'avril 1960, a pris fin lors du coup d'État de 1961. Dès lors stigmatisée dans le camp de l'opposition au régime militaire dirigé par Chung-Hee Park, l'Église catholique ne pouvait qu'être freinée dans son développement6.

2. Werner Sasse, « Minjung Theology and Culture », Papers of the British Association for Korean Studies, vol. 1, ISSN 09651942, p. 29-43.

3. La fin de l'ère confucéenne a permis à cette religion interdite de reprendre vie et de s'installer à nouveau dans les villes. Les bouddhistes ont alors imité les protestants, louant des salles dans les mêmes immeubles qu'eux, créant un culte dominical, traduisant en coréen les chants et les prières, qui n'étaient jusqu'alors disponibles qu'en chinois classique, et les accompagnant même au piano.

4. Exposé d'Évelyne Chérel pour les conférences du Réseau Asie, 24 et 25 septembre 2003, « Les Églises chrétiennes et l'État en Corée du Sud (1945-1950) : un lien de type symbiotique ». Panel 19: «Vers un lien symbiotique entre le politique et le religieux ». Disponible sur www.reseau-asie.com.

5. Ibid., p. 4.

6. É. Chérel, «Politique et religion en Corée du Sud : Jeunesse catholique et les élections générales de mai 1948. Un exemple de politisation d'une revue catholique », thèse de doctorat en lettres, Paris VII, UFR Langues et civilisations d'Asie orientale, 1999, p. 10-17. 


\section{Élaboration d'une première théodicée mobilisatrice en milieu protestant}

Lorsqu'il a été introduit en Corée à la fin du XIXe siècle, le protestantisme a bénéficié d'un siècle de revendications catholiques auprès des puissances occidentales, revendications qui avaient abouti à ce que les États-Unis en 1882, puis la France en 1886 posent la garantie d'une plus grande liberté religieuse comme condition indispensable à la signature des premiers traités commerciaux avec la Corée. Le climat politique était, de ce fait, plus ouvert à l'Occident. Parallèlement, l'élite confucéenne commençait à être contestée, non seulement par les gens du peuple, mais aussi par les nobles du Nord-Ouest coréen, qui cherchaient à imposer leur volonté de réformes. C'est donc précisément dans cette région que le protestantisme s'est développé le plus rapidement. Les réformateurs ont été sensibles aux notions d'égalité qu'ils découvraient dans la doctrine chrétienne et dont ils voyaient la concrétisation dans la construction d'écoles et d'hôpitaux pris en charge par les missionnaires protestants américains. Il leur est apparu très vite que la nouvelle religion était la seule voie d'accès à la modernité.

Le sociologue Chung-Shin Park observe que la croissance du protestantisme commence dès1894, au moment de la guerre sino-japonaise, soit dix ans seulement après l'arrivée des premiers missionnaires sur la péninsule. Cette guerre, qui a détruit l'ordre sinocentrique de l'Asie orientale et permis au Japon de prétendre au leadership de la région, a mis fin à la dépendance culturelle et politique de la Corée visà-vis de la Chine qui durait depuis plusieurs siècles. Elle a profondément impressionné les Coréens, convaincus désormais que les Japonais tenaient leur supériorité de la connaissance des valeurs et pratiques occidentales7. Privés de toute autre civilisation de référence, ils se sont alors tournés vers la puissance états-unienne et ont vu dans le protestantisme la manifestation d'une civilisation capable de les protéger de l'ambition hégémonique des Japonais.

Paradoxalement, le Memorandum Taft-Katsura (1905) n'a pas altéré l'image positive que les Coréens se faisaient des États-Unis. Par ce pacte secret, le président Théodore Roosevelt approuvait pourtant la colonisation de la Corée par le Japon qui, en échange, s'engageait à ne pas intervenir dans les affaires américaines aux Philippines. De façon tout aussi paradoxale, la non-intervention des missionnaires protestants étrangers dans la politique du gouvernement colonial n'a pas ébranlé l'espoir que les Coréens plaçaient dans la nouvelle religion.

Pour comprendre le succès du protestantisme - qu'il soit méthodiste, presbytérien, baptiste, pentecôtiste, évangélique, conservateur ou libéral -, il faut avant tout tenir compte de sa grande plasticité, qui, dès le départ, a permis aux Coréens de s'approprier cette religion étrangère. On retrouve d'ailleurs ce processus d'indigénisation en Afrique ou en Amérique latine, et ce chaque fois qu'un pays traverse une période de crise qui menace de le faire basculer dans une situation de profonde 
anomie. L'orientation messianique-millénariste que les Coréens ont donné à leur lecture de la Bible est caractéristique de la «théodicée », telle que la définit notamment Peter Berger, c'est-à-dire un « édifice de significations englobant toute chose » et élaborant une remise en ordre du mondes. Lorsque le Japon a colonisé la Corée en 1910, de nombreux Coréens, frustrés et humiliés, ont reporté tous leurs espoirs dans la seconde venue du Christ9. Cette attente du Jour Glorieux leur a permis de relativiser leurs souffrances et de supporter l'injustice tout en leur donnant les arguments pour réintégrer l'histoire coréenne dans un ordre universel10. En personnifiant le Japon sous la figure de Satan et en présentant la tragédie de la colonisation comme le signe de la fin des temps, la doctrine chrétienne fondée sur l'opposition du Bien et du Mal a donné sens au projet politique de libération du peuple coréen.

Si cette théodicée a pu être largement mobilisatrice, c'est parce qu'il existait des communautés suffisamment structurées pour assurer sa diffusion. Du point de vue social, le réseau protestant était le réseau le mieux organisé de tout le pays. Les églises publiaient de nombreux journaux et magazines traitant de thèmes aussi bien religieux que sociaux et des centaines d'établissements scolaires et universitaires privés étaient dirigés par des protestants11. C'est dans ces établissements qu'ont été formés les leaders politiques coréens qui ont remplacé les missionnaires aux postes de responsabilités administratives et ont transformé ces églises en relais de communication parfaitement rodés à travers toute la Corée. C'est en cela qu'il y a eu indigénisation des Églises. La position antijaponaise de la communauté protestante lui a valu la sympathie et la confiance des Coréens colonisés, et ce sont cette confiance et cette sympathie qui ont favorisé son essor en attirant tous ceux qui voulaient s'engager dans le mouvement de résistance à la colonisation japonaise. L'Église protestante était alors « la plus grande, sinon l'unique communauté coréenne organisée : tout à la fois lieu de consolation, forum politique, réseau de communication et base pour l'organisation des activités sociales et politiques. Grâce à cette mobilisation, [elle a continué] de se développer rapidement, et [est devenue] au cours de cette période, la vraie religion coréenne »12. Durant toute la première décennie de la colonisation, elle a été à l'origine de la construction idéologique d'un « nous », fortifié par une expérience collective inédite. Pour la première fois de leur histoire, des Coréens partageaient une même foi - aussi

\footnotetext{
7. Chung-Shin Park, Protestantism and Politics in Korea, Seattle, University of Washington Press, 2003, p. 24.

8. Peter L. Berger, La religion dans la conscience moderne : essai d'analyse culturelle, traduit de l'anglais par Joseph Feisthauer, Paris, Éditions du Centurion, 1971, p. 97.

9. Chung-Shin Park, Protestantism and Politics in Korea, op. cit, p. 61.

10. P. L. Berger, La religion dans la conscience moderne..., op. cit., p. 119-120.

11. Ibid., p. 33.

12. Chung-Shin Park, Protestantism and Politics in Korea, op. cit, p. 129 (nous traduisons).
} 
bien patriotique que religieuse - et se réunissaient durant des jours et des nuits pour prier ensemble.

Il était donc logique que les protestants fussent largement impliqués dans la Déclaration d'Indépendance de 1919. Cependant, la forte répression qui a suivi la manifestation pacifique du 1er mars a brisé l'espoir qu'ils avaient largement contribué à entretenir: le mal avait fini par vaincre, réduisant à néant les prophéties de la théodicée. Durant les vingt dernières années de la colonisation, le mouvement de résistance s'est organisé en dehors des frontières, tandis que dans le pays les Églises ont dû fonctionner, à l'instar des autres religions (bouddhiste, confucéenne et catholique), comme une institution établie, soumise à l'ordre japonais. La mémoire de ce « nous » revalorisé n'a pas été perdue pour autant. Après la colonisation, les lieux de culte qui n'avaient pas disparu des villes et particulièrement de Séoul ont été à nouveau investis et ont permis de relancer la croissance du protestantisme. Si le Japon avait été chassé, un autre mal émergeait, le communisme, qui redonnait toute son efficacité mobilisatrice à la symbolique traditionnelle du Bien et du Mal.

\section{Le rôle centralisateur des Églises conservatrices en Corée du Sud}

L'urbanisation accélérée que connaît la Corée du Sud dans les années 1950 fournit aux Églises chrétiennes, toutes dénominations et tendances confondues, une nouvelle occasion d'exercer leur influence. Cette fois, ce sont des populations bouleversées par la disparition du schéma familial traditionnel, provoquée par l'exode rural, qui recherchent de nouvelles structures communautaires. Les Églises sont à nouveau perçues comme un lieu protecteur, un refuge contre la détresse. Elles attirent tout particulièrement les jeunes filles venues de la campagne et effrayées par la solitude et les dangers de la vie citadine13. Très rapidement, les Églises protestantes ont à nouveau l'avantage par rapport à l'Église catholique, grâce aux dons importants qu'elles reçoivent des Églises mères américaines. Cet argent leur permet d'infiltrer la sphère publique, notamment dans les domaines de la santé et de l'éducation, plus ou moins délaissés par l'État. Outre les écoles secondaires et les universités privées, les orphelinats, les maisons de retraites ou d'accueil pour les handicapés sont autant de lieux investis par le religieux. Cette absence de distinction entre institutions religieuses et sociales permet de comprendre les raisons très pragmatiques que les Coréens donnent de leur conversion. Ce peut être le désir d'entrer dans tel lycée privé dirigé par les Églises, le besoin de trouver une structure d'accueil pour un enfant handicapé ou, plus simplement, le souhait d'être intégré dans un milieu social particulier. La conversion est avant tout suscitée non par une sensibilité religieuse (qui peut exister par ailleurs) mais par la possibilité qu'elle offre d'accéder à telle structure médicale, éducative ou sociale, qui, autrement, serait inaccessible14. Cette indifférenciation institutionnelle a favorisé des modalités 
d'adhésion radicalement différentes de celles que la modernité occidentale, fondée sur le principe de sécularisation de la société, suscite sur le terrain religieux. Ainsi, tandis que Grace Davie fait du believing without belonging un des paramètres de la modernité religieuse désinstitutionnalisée, les témoignages des convertis coréens illustrent le paramètre inverse, belonging without believing, selon lequel ce qui compte avant tout ce sont les liens institutionnels structurant l'appartenance à l’Église15.

\section{La Corée du Sud réenchantée}

Outre cette mainmise sur différents secteurs sociaux, les courants protestants fondamentalistes et conservateurs ont pu accélérer leur croissance grâce au soutien politique qu'ils offraient aux gouvernements militaires qui se sont succédé jusqu'en 1992, date de l'arrivée au pouvoir d'un président civil, Yong-Sam Kim. Dès la fin de la seconde guerre mondiale, la présence soviétique au Nord obligea les nombreux protestants originaires de la région du Nord-Ouest à migrer massivement vers le Sud. Cette migration donna corps à un nouveau dualisme symbolique qui opposait cette fois le Nord, sous influence satanique, au Sud, élu par Dieu. Cette théodicée, largement partagée par l'ensemble des Églises, ne les a pas toutes conduites à placer au second plan la nature du régime au pouvoir. C'est ce qui a distingué l'ensemble des courants fondamentalistes - dont la courbe de croissance a été très positive -, des courants catholiques et libéraux - qui sont restés minoritaires. Pour les premiers, seule importait la lutte contre le communisme. C'est pour cette raison qu'ils ont légitimé la politique plus qu'autoritaire de l'État militaire coréen et sacralisé son autorité comme divinement inspirée. Selon cette logique, tous ceux qui s'écartaient des programmes définis par le gouvernement étaient des traîtres qui se laissaient attirer par les forces du Mal.

Deux nouveaux mouvements indigènes ont poussé cette rhétorique à son paroxysme : l'Église messianique de l'Unification fondée en 1953 par le révérend Sun-Myông Moon (originaire de la Corée du Nord) et l'Église pentecôtiste prophétique du Plein Évangile fondée à la même époque par le révérend Yonggi Cho. En faisant de la Corée du Sud, traumatisée par la guerre et la division, le pays élu, ces deux Églises ont donné à l'État sud-coréen une puissante légitimation religieuse.

\footnotetext{
13. Voir Robert F. Spencer, Yôgong : Factory Girl, Séoul, Royal Asiatic Society, Korea Branch, 1988 et Uchông Li, « Yôsông nodongjadûlgwa minjungwa-undong » (Le mouvement populaire et ouvrier féminin), Hyôndae Hanguk Yôsôngnon, (Les femmes dans la Corée d'aujourd'hui), Samminsa, 1986.

14. Conclusion des analyses d'interviews menées durant la décennie 1990 dans l'ensemble des dénominations chrétiennes.

15. Yôngae Ch'oe, « Yôsônggwa chonggyo. Kaesingyo kyohoe chubu sindolûl chungsimûlo » (Les femmes et la religion. Les femmes au foyer comme fidèles des Églises renouvelées), Tto hanaûi munhwa (Une autre culture), 6, 1990, p. $294-307$.
} 
Elles ont notamment réussi à intégrer dans leur message le projet politique sudcoréen cristallisé, dans le contexte international de la guerre froide, autour du 38e parallèle, la ligne de séparation des deux Corée. La doctrine messianique de Moon fait de celui-ci l'initiateur de la troisième et dernière période du projet divin : après l'Ancien, puis le Nouveau Testament, doit venir le « Testament accompli », dont l'interprétation de l'histoire de l'humanité fait du communisme l'ennemi de Dieu et la manifestation moderne de l'esprit de Caïn. Parallèlement, le capitalisme, confondu avec la démocratie, est associé à la sagesse d'Abel. La lutte des deux frères est représentée par la guerre froide. Dans l'Ancien Testament, Caïn a tué Abel et le Mal a régné sur la terre. Le Testament accompli annonce la destruction de Caïn et la restauration du paradis terrestre. La victoire doit se concrétiser par la chute des dirigeants de la Corée du Nord et la réunification des deux Corée en un pays capitaliste. La propagande anticommuniste de l'Église de l'Unification a été d'autant plus puissante qu'elle a réussi à séduire des Occidentaux (également concernés par la guerre froide, mais aussi en quête de sens) et à gagner la confiance de quelques personnalités politiques américaines pour qui le révérend Moon est devenu le représentant privilégié de la Corée du Sud. Le soutien américain s'est cependant fortement atténué lorsqu'il s'est avéré que, s'il prenait bien les États-Unis pour modèle, Moon entendait surtout en faire le tremplin qui pourrait propulser la Corée au centre du monde16.

L'ambition politique de Moon a fini par gêner aussi le gouvernement sudcoréen. En 1976, il a retiré leurs passeports aux Petits Anges, la célèbre troupe de jeunes danseurs moonistes, privant par là même l'Église d'une source importante de revenus et d'un moyen de reconnaissance internationale. En 1977, le gouvernement a également poursuivi et arrêté les cadres de l'entreprise pharmaceutique mooniste, IlHwa, par l'intermédiaire de laquelle s'opéraient d'importants transferts de fonds au profit de l'association cultuelle. En revanche, la conduite plus réservée, mais tout aussi nationaliste et progouvernementale, de Yonggi Cho a permis à son Église d'être, en 1980, la première institution religieuse acceptée sur l'île de Yôuido, où siège l'Assemblée nationale. Les bâtiments abritant l'Église et l'Assemblée nationale sont construits face à face, à quelques kilomètres à peine l'un de l'autre, et dans un même style architectural, pourtant peu courant en Corée du Sud.

Le fait d'accorder à une église protestante le droit de s'installer dans un lieu qui est symboliquement le cœur de la vie politique est révélateur des liens étroits que les gouvernements ont entretenus avec les groupes religieux anticommunistes. Sous la présidence de Syngman Rhee, le gouvernement réunissait des prédicateurs, des députés, des hauts fonctionnaires et des hommes d'affaires à l'occasion de petits déjeuners de prières, auxquels participaient parfois le président lui-même et le Premier ministre. La Corée a alors tout d'un État chrétien : le président prête serment sur la Bible au cours de la cérémonie de prise de fonction, le jour de Noël est décrété 
jour férié et les programmes d'évangélisation sont diffusés par la radio d'État17.

Parce que le pays a été divisé et qu'il a fallu constamment donner sens à cette déchirure, à cette séparation des destins, la théodicée anticommuniste a été le facteur de mobilisation, qui, jusque dans les années 1990, a évité à la Corée du Sud d'être perdue dans ce que Patrick Michel appelle l' « ère du relatif ». En effet, « l'existence du système soviétique [ayant] conduit à une idéologisation de la démocratie constituée en espace d'incarnation du Bien face à l'empire du Mal », l'effondrement de l'Union soviétique a eu tendance à supprimer la super-référence - l'ennemi -, qui permettait de se définir et de produire un sens socialement et politiquement mobilisateur18. Mais, dans un pays encore aujourd'hui déchiré, la superréférence ne disparaît pas aussi vite. La Corée du Sud s'est maintenue dans une vision enchantée de son histoire, qui l'a préservée d'une modernisation à l'occidentale marquée par le désenchantement du monde et l'individualisation des destins. Jusqu'à ce que la crise asiatique mette définitivement à mal les fondements d'une théodicée qui faisait de la réussite économique sud-coréenne le signe de la prédilection divine, la progression de la Corée du Sud sur la scène économique internationale a été l'enjeu d'une mobilisation sans faille. Les Églises protestantes fondamentalistes ont servi à convaincre le peuple d'accepter les sacrifices qu'exigeait le redressement du pays. Cela a sans aucun doute facilité la fulgurante croissance économique de la Corée du Sud (plus de 8 \% par an de 1960 à 1980)19. C'est donc au niveau économique que ces Églises ont joué un rôle déterminant dans la modernisation de ce pays. On peut y voir l'une des raisons du succès, national et international, de mouvements aussi ouvertement portés sur la réussite économique que l'était - et l'est encore plus modestement - l'Église du Plein Évangile pour laquelle la promesse de réussite valait tout autant pour le pays que pour l'individu.

\section{Le tournant des années 1980}

La progression économique de la Corée du Sud ralentit cependant à partir des années 1980, période d'intenses révoltes des Coréens contre la politique autoritaire du gouvernement. En octobre1979, le président Chung-Hee Park, au pouvoir depuis 1961 est assassiné. Contrairement aux espoirs du peuple, son successeur,

\footnotetext{
16. Dans ces conditions, il n'est pas étonnant que le rapport américain publié en 1978 sur l'Église de l'Unification ait été très négatif. Cf. Investigation of Korean-American Relations, Report of the Subcommittee on International Organizations of the Committee on International Relations U.S. House of Representatives, Washington, 31 octobre 1978.

17. Bertrand Chung, «Politique et religion en Corée du Sud », Revue d'études contemporaines Est-Ouest, 32 (1), 2001 , p. 92.

18. Patrick Michel, Politique et religion. La grande mutation, Paris, Albin Michel, 1994, p. 36.

19. Mario Lanzarotti, La Corée du Sud : une sortie du sous-développement, Paris, PUF, coll. « Tiers Monde », 1992.
} 
Du-Hwan Chun, instaure la cinquième République sans rien changer aux modalités d'exercice du pouvoir. De février à mai 1980, les grèves d'ouvriers et les manifestations d'étudiants se multiplient. Les Coréens veulent de meilleurs salaires, plus de loisirs, mais surtout plus de démocratie. C'est le fameux «printemps de Séoul » qui s'achève par une répression sanglante.

Certains courants chrétiens modifient alors leur stratégie de mobilisation et s'orientent plus précisément vers l'encadrement individuel. L'Église du Plein Évangile construit un centre de missions qui a pour vocation de répondre simultanément aux besoins professionnels, sociaux et familiaux de ses fidèles. Ces missions sont de remarquables réseaux organisés par entreprise et par catégorie professionnelle - médecins, militaires, policiers, coiffeurs, athlètes, enseignants, ouvriers, ingénieurs, etc. ; sociale - personnes âgées (l'Église a sa propre maison de retraite), célibataires (elle a également son agence matrimoniale), enfants, jeunes, étudiants, handicapés, etc., ou encore culturelle20.

Les adhérents évoluent dans ces missions en fonction de leur parcours individuel. Ils y trouvent ce que l'on pourrait appeler un accompagnement personnalisé. Ainsi, la possibilité qu'ont des salariés de même statut, mais d'entreprises différentes, d'établir des liens grâce à ces structures permet aux Églises d'assurer leur solidité sur le long terme. Elles favorisent la formation de groupes corporatistes à l'intérieur desquels la solidarité permet de trouver la solution à des problèmes d'ordre professionnel. Face à la faible influence, à cette époque, des syndicats, ces regroupements sont très efficaces et ne cessent d'attirer de nouveaux membres à l'intérieur de chaque corporation, d'autant que, d'un bureau à l'autre, les rencontres à différents niveaux hiérarchiques permettent un traitement de faveur entre chrétiens d'une même dénomination21. Parallèlement, ces structures permettent de calmer l'esprit de révolte des pratiquants qui se sentent mieux suivis individuellement. Elles accompagnent ainsi le travail de reprise en main de Du-Hwan Chun.

Selon la même volonté d'adaptation aux nouvelles revendications sociales, un nouveau groupe est formé en 1980 par Myông-Sôk Jông (Jông Myông-Sôk dit JMS: Jesus Morning Star), en rupture avec l'Église de l'Unification. JMS récupère la doctrine de Moon, tout en étant beaucoup plus discret sur le registre de l'anticommunisme de son message. Le mouvement se développe dans les milieux universitaires, à la faveur de rencontres dans les stades, les piscines ou les théâtres. Il transforme d'ailleurs le culte dominical en matchs de football qui se déroulent sur les campus et dont l'interprétation se fait, malgré tout, sur fond de doctrine anticommuniste de Moon : les équipes représentent le Bien et le Mal, le capitalisme et le communisme, la Corée du Sud et la Corée du Nord. Les vainqueurs sont toujours du côté de JMS. La coloration sportive du message permet au groupe de soutenir les préoccupations gouvernementales de l'époque : en effet, mobiliser les étudiants autour d'un enjeu sportif devient une façon de participer à la prépara- 
tion générale des jeux olympiques de Séoul de 1988. On en revient ainsi, une fois de plus, au constat que ces mouvements progouvernementaux ont exploité les crises et donner un sens enchanté à l'histoire de la Corée du Sud. Myông-Sôk Jông est parvenu à séduire des étudiants qui s'étaient fortement engagés dans les tentatives révolutionnaires avortées de 1980. Il leur a permis de surmonter le vide laissé par leur échec tout en les soumettant au régime en place. Il est surprenant de voir que ces mouvements messianiques, qui ont fleuri par centaines, ne proposent pas un renouvellement du monde, comme ils peuvent le faire dans d'autres aires culturelles. Ici, ils donnent un sens à l'ordre qui règne et légitiment le pouvoir en place pour rendre plus douce la soumission aux contraintes qu'il impose.

L'adaptation des Églises progouvernementales aux revendications des Coréens du Sud des années 1980 s'est opérée selon une volonté de mobilisation et de centralisation semblable à celle des premières Églises apparues trente ans plus tôt. Loin de contribuer à l'amorce des processus de différenciation des institutions, d'individualisation des destins et de désenchantement caractéristiques de la modernité entendue dans son acception occidentale, ces puissantes machines institutionnelles ont cumulé les fonctions culturelle, religieuse, politique, économique et sociale, pour mieux se centrer sur une priorité unique : vaincre le communisme de la Corée du Nord en affichant la supériorité du capitalisme du Sud.

\section{L'entrée de la Corée du Sud dans la modernité et la fin de l'expansion des protestantismes}

La crise asiatique de 1997 a remis en cause le postulat de l'opposition Nord/Sud. La preuve de l'élection divine ayant été entièrement placée sous le signe de la réussite économique, cette crise a brutalement brisé la capacité de la théodicée à faire sens. La Corée s'est retrouvée soudainement désenchantée. Les Églises conservatrices ont tenté un dernier effet. En vain. Leur nouveau message allait trop à l'encontre de la logique d'enrichissement collectif et individuel assénée depuis près d'un demi siècle. À la veille du nouveau millénaire, leurs sermons se sont faits menaçants, insistant sur la colère de Dieu qui punissait les Coréens du Sud pour leur ingratitude. Dieu leur avait beaucoup donné, mais puisqu'ils avaient oublié leur Sauveur, il n'était plus question de bienfaits matériels : désormais, il fallait économiser. Jusqu'en 1999, ce discours a été diffusé à tous les niveaux de la chaîne prosélyte, dans les églises mais aussi dans la rue ou le métro22. Mais après l'an 2000,

\footnotetext{
20. À cette même période, l'Église du Plein Évangile s'organise également au niveau mondial : des missions sont ouvertes en Asie, en Afrique, ou en Amérique latine. L'Église devient un modèle pour de nombreux pays en voie de développement. 21. Voir Nathalie Luca, «Pentecôtismes en Corée », Archives de sciences sociales des religions, 105, janvier-mars 1999, p. 99-123. 22. Observations de terrain, hiver 1999.
} 
la chaîne s'est brisée, comme si cette année butoir, où rien de miraculeux ne s'est produit, marquait définitivement la fin de l'exception sud-coréenne. La Corée du Sud a dès lors cessé de prétendre au titre de pays élu. Les interprétations théologiques ont perdu le zèle nationaliste qui avait fait la force des Églises protestantes depuis le début du siècle dernier. Elles se limitent désormais à un message individualiste plutôt fade et craintif : «On se sent moins seul quand on est près de Jésus »23.

Certes, personne n'aurait l'idée d'envier la situation de la Corée du Nord, mais il n'en reste pas moins qu'aujourd'hui le Sud ne se porte pas aussi bien qu'on voudrait le faire croire. L'efficacité du modèle capitaliste américain est remise en question et l'on s'interroge sur la bonne volonté des États-Unis dont l'ingérence dans les affaires nationales, qu'elle soit militaire ou économique, est fortement critiquée. Les manifestations anti-américaines se multiplient et sont renforcées par l'engagement des militaires sud-coréens dans la guerre contre l'Irak à laquelle le peuple était opposé. À cela s'ajoute la réelle démocratisation du gouvernement sudcoréen qui permet le renouveau de groupes jusque-là fortement surveillés et ouvre la Corée du Sud à un marché religieux international. La démocratisation du régime permet également une réelle sécularisation des institutions. Les syndicats indépendants se développent et la protection sociale peut être découplée de l'adhésion religieuse. Enfin, alors que la réunification de l'Allemagne et la chute du communisme dans l'ex-Union soviétique avaient déjà contribué à affaiblir le nationalisme anticommuniste, l'option politique de réconciliation avec le Nord le rend obsolète.

En quelques années, la Corée du Sud est finalement devenue une société moderne, c'est-à-dire une société qui vit « dans une condition d'incertitude structurelle »24, où le doute est dans l'esprit de chacun et où le recours collectif au miraculeux est inopérant. Sans le soutien paradoxal d'un ennemi à combattre, les Coréens se sont démobilisés et chacun cherche désormais le moyen de donner sens à sa propre existence. Ce nouveau contexte explique aussi bien le changement de ton des prédicateurs protestants qui insistent désormais sur le bien-être et le salut individuels sans autres prétentions, que la progression notable de nouveaux mouvements religieux dont certains sont hostiles au capitalisme. C'est le cas du falungong, une association fortement réprimée en Chine et critiquée par les médias coréens, qui aurait fait en trois ans plus de 10000 adhérents25. Au-delà de cette estimation qu'il est difficile de vérifier, ce sont les modalités d'adhésion, fort peu contraignantes, radicalement opposées en tout cas à celles des courants chrétiens dont la croissance est désormais stoppée, qui retiennent l'attention: on se retrouve, le matin ou le soir, dans les parcs, pour pratiquer une forme de yoga en musique; on échange à peine quelques mots avec les autres pratiquants, puis l'on se sépare. C'en est fini des grands rassemblements et des discours mobilisateurs. Il ne reste plus qu'une pratique individuelle, seule capable de donner sens à un destin unique, et qui ne semble pas avoir vocation à être partagée ailleurs que sur internet. Les 
adeptes les plus convaincus assimilent les principes du fondateur Li Hongzhi chez eux, dans la solitude, avec une grande rigueur. Les autres sont simplement attirés par la pratique yoggique26. Une grande variété de nouveaux mouvements religieux, de type New Age et hostiles au protestantisme, emportent désormais l'adhésion de couches sociales très diverses qui n'acceptent plus qu'une religion puisse prétendre diriger leur existence dans toutes ses dimensions. La vie professionnelle des adeptes du falungong est d'ailleurs souvent en contradiction avec les principes de la doctrine. Face au désarroi idéologique, on observe un retour à des conceptions bouddhistes du destin, fondées notamment sur la croyance que l'individu ne peut se transformer que par ses propres moyens. Les multiples écoles de gigong, d'origine chinoise, dont fait partie le falungong, profitent de ce changement de mentalités, comme si, déçus par le modèle occidental dans lequel ils avaient mis toute leur foi, les Coréens du Sud revenaient à leur civilisation de référence, la Chine, au moment même où celle-ci fait retour sur son propre passé.

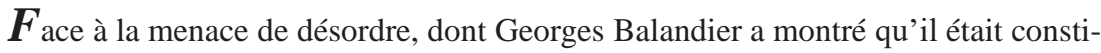
tutif de la modernité, la force des protestantismes, en Corée, a été précisément d'assurer la stabilité sociale, et ce au détriment de la modernisation du pays. Ces mouvements sont parvenus à conjurer les risques d'anomie durant la colonisation et après la guerre. Mais le dualisme symbolique, formé dès avant 1948, a aussi contribué à provoquer la scission entre le Nord et le Sud. La croissance économique, individuelle et nationale, a été mise au service de la thèse globale du Bien capitaliste luttant contre le Mal communiste. La pauvreté de la Corée du Nord est devenue a contrario la preuve logique de l'élection de la Corée du Sud. Cette projection a permis d'intérioriser l'ordre social, certes insatisfaisant mais vécu par beaucoup comme inévitable et protecteur. En même temps qu'elles enchantaient la Corée du Sud, les institutions fondamentalistes protestantes ont occupé la sphère publique, en investissant les domaines de la santé et de l'éducation ou en soutenant le gouvernement. Au moment même où, en Occident, le processus de sécularisation était en cours, soustrayant à l'autorité religieuse des secteurs entiers de la vie sociale et culturelle, en Corée du Sud, ces secteurs se structuraient sous le contrôle des Églises protestantes. L'individu, quant à lui, encadré dans tous les domaines de sa vie publique et privée, voyait son destin lié de manière indissoluble à celui de son pays. La mise en ordre des mondes et le maintien de leur stabilité sont donc

23. Observations de terrain, printemps 2003.

24. Danièle Hervieu-Léger, « Croire en modernité : au-delà de la problématique des champs religieux et politiques », dans

P. Michel (dir.), Religion et démocratie, Paris, Albin Michel, 1997, p. 372.

25. Chiffre fourni par les adeptes eux-mêmes.

26. Étude ethnographique réalisée au printemps 2003. 
bien passés par une impossibilité de l'État sud-coréen à moderniser ses structures sociétales. La crise asiatique a bouleversé la donne en introduisant le désordre. Elle s'est imposée comme un fait social inéluctable devant lequel s'effondrait la crédibilité de la théodicée. Elle a introduit des paramètres d'incertitude et de désorientation et bouleversé « les systèmes de valeur et de repérage, les cadres et les dispositifs inconscients qui règlent la quotidienneté »27. Cette désorientation a été accentuée tant par le regard critique porté sur les États-Unis que par la volonté politique de réconciliation avec la Corée du Nord. L'effondrement de l'ensemble des valeurs jusqu'alors portées par les Églises protestantes les a brusquement vidées de leur contenu et par là même de leur raison d'être. La Corée du Sud se retrouve désormais en phase avec les pays occidentaux. Les processus de démocratisation, de sécularisation et d'individualisation se poursuivent en même temps que s'achève toute prétention à dire ce que doit être l'ordre. I

Nathalie Luca est chargée de recherches au CNRS-EHESS (Centre d'études interdisciplinaires des faits religieux). Elle a publié dernièrement Le salut par le foot. Une ethnologue chez un messie coréen (Genève, Labor et Fides, 1997) ; Sectes, mensonges et idéaux, (avec Frédéric Lenoir, Paris, Bayard, 1998) ; «Pentecôtismes en Corée », Archives de sciences sociales des religions (105, janvier-mars 1999, p. 99-123) ; " La conquête de la modernité par les nouveaux mouvements chrétiens coréens », Social Compass (47 (4), 2000, p. 525-539) et «Quelles politiques face aux sectes ? ", Critique internationale (17, octobre 2002, p. 105-125). Email : luca@ehess.fr

27. Georges Balandier, Le détour : pouvoir et modernité (1985), Paris, Fayard, 1997, p. 14 